\title{
ON THE SPARSITY ORDER OF A GRAPH AND ITS DEFICIENCY IN CHORDALITY
}

\author{
MONIQUE LAURENT
}

Received August 31, 1998

Revised April 26, 2000

\begin{abstract}
Given a graph $G$ on $n$ nodes, let $\mathcal{P}_{G}$ denote the cone consisting of the positive semidefinite $n \times n$ matrices (with real or complex entries) having a zero entry at every off-diagonal position corresponding to a non edge of $G$. Then, the sparsity order of $G$ is defined as the maximum rank of a matrix lying on an extreme ray of the cone $\mathcal{P}_{G}$.

It is known that the graphs with sparsity order 1 are the chordal graphs and a characterization of the graphs with sparsity order 2 is conjectured in [1] in the real case. We show in this paper the validity of this conjecture. Moreover, we characterize the graphs with sparsity order 2 in the complex case and we give a decomposition result for the graphs with sparsity order $\leq 2$ in both real and complex cases. As an application, these graphs can be recognized in polynomial time.

We also indicate how an inequality from [17] relating the sparsity order of a graph and its minimum fill-in can be derived from a result concerning the dimension of the faces of the cone $\mathcal{P}_{G}$.
\end{abstract}

\section{Introduction}

In this paper we study the ranks of extremal positive semidefinite matrices with a given sparsity pattern, in continuation of the papers [1], [5], [6], [7], [12], [13], [17]. This study is motivated mainly by its application to the completion problem for positive semidefinite matrices (details are given below) and it is also relevant to chordal graphs and Gaussian elimination for sparse positive definite matrices ([15]).

Mathematics Subject Classification (2000): 05C50, 05C75, 15A48, 15A57. 
The sparsity order of a graph. Let $G=(V, E)$ be a graph with node set $V=\{1,2, \ldots, n\}$ and let $\bar{E}$ denote the set of non edges; that is, $\bar{E}$ is the set of pairs $i j$ with $i \neq j \in V$ and $i j \notin E$. Let $\mathcal{P}_{G}$ denote the set of positive semidefinite $n \times n$ matrices whose $i j$-th entries are zero for all $i j \in \bar{E}$. All matrices are assumed to have entries in the field $\mathbb{F}$, where $\mathbb{F}$ is equal to $\mathbb{R}$ (the field of real numbers) or $\mathbb{C}$ (the field of complex numbers). The set $\mathcal{P}_{G}$ is a closed convex cone. A matrix $X \in \mathcal{P}_{G}$ is said to be extremal if it lies on an extreme ray of the cone $\mathcal{P}_{G}$. The sparsity order of $G$ (also abbreviated as the order of $G$ ) $\operatorname{ord}_{\mathbb{F}}(G)$ is defined as the maximum rank of an extremal matrix in $\mathcal{P}_{G}$. We have: $\operatorname{ord}_{\mathbb{F}}(G) \leq n-2$, with equality in the real case when $n \geq 4$ if and only if $G$ is a circuit [7]. When $G$ is the complete graph, $\mathcal{P}_{G}$ is the cone of all positive semidefinite matrices, every extremal matrix has rank $\leq 1$ and, thus, $\operatorname{ord}_{\mathbb{F}}(G)=1$. The sparsity order of a complete bipartite graph is computed in [5] and the graphs with sparsity order 1 are characterized in [1] (cf. also [14]). Namely,

Theorem 1. For a graph $G$, we have: $\operatorname{ord}_{\mathbb{R}}(G)=1 \Longleftrightarrow \operatorname{ord}_{\mathbb{C}}(G)=1 \Longleftrightarrow G$ is chordal, i.e., $G$ does not contain any circuit of length $\geq 4$ as an induced subgraph.

The following two operations are useful for computing the sparsity order. If $H$ is an induced subgraph of $G$, then

$$
\operatorname{ord}_{\mathbb{F}}(H) \leq \operatorname{ord}_{\mathbb{F}}(G)
$$

[1] and if $G$ is the clique sum of two graphs $G_{1}$ and $G_{2}$, then

$$
\operatorname{ord}_{\mathbb{F}}(G)=\max \left(\operatorname{ord}_{\mathbb{F}}\left(G_{1}\right), \operatorname{ord}_{\mathbb{F}}\left(G_{2}\right)\right)
$$

[7]. A graph $G$ is called a $k$-block if $G$ has order $k$ and every proper induced subgraph of $G$ has order $\leq k-1$. For instance, the circuit $C_{n}$ is an $(n-2)$ block over the reals if $n \geq 4$ [1]. For any given $k$, the number of $k$-blocks is finite, because a $k$-block has at most $k^{2}+k-2$ (resp. $k^{2}-1$ ) nodes if $\mathbb{F}=\mathbb{R}($ resp. $\mathbb{F}=\mathbb{C})[1]$. In both the real and complex cases, $K_{1}$ is the only 1-block and $C_{4}$ is the only 2-block; in the real case, there are exactly sixteen 3-blocks: the graphs $A_{1}-A_{10}$ and $B_{1}-B_{6}$ shown in Figure 2. [1]. Helton et al. [6] have classified the 4-blocks over $\mathbb{R}$ having 9 non edges (by relation (5), this is the smallest number of non edges that a 4-block can have); their number is quite large and the classification involves many technical details. This indicates the difficulty of the general problem of classifying $k$-blocks.

In order to characterize the graphs having order $\leq k$, it suffices to know the minimal (with respect to taking induced subgraphs) graphs among the $p$ blocks with $p>k$ and this might be more tractable, at least for small values 
of $k$. For instance, it follows from Theorem 1 that every $k$-block $(k \geq 2)$ contains a circuit of length $\geq 4$ as an induced subgraph. The following is conjectured in [1] in the case $k=2$ and $\mathbb{F}=\mathbb{R}$ :

Conjecture 2. A graph $G$ satisfies: $\operatorname{ord}_{\mathbb{R}}(G) \leq 2$, if and only if $G$ does not contain as an induced subgraph a circuit on $n \geq 5$ nodes nor a 3-block. Equivalently, over the reals, the only $k$-block $(k \geq 4)$ which contains no 3 block is the circuit $C_{k+2}$.

The main contribution of this paper is to show the validity of Conjecture 2 (cf. Theorem 9); we also prove an analogous characterization for the graphs having sparsity order $\leq 2$ over $\mathbb{C}$ (cf. Theorem 13). The essential ingredient in our proof is a decomposition result (in terms of clique sums) for the class of graphs having no 3-block and no circuit of length $\geq 5$ as an induced subgraph (cf. Theorem 8). Section 5 is devoted to the proof of this result which is quite technical.

As an application, one can recognize in polynomial time whether a graph has order 1 or 2 . As another application, we can characterize the graphs whose 'powers' all have order $\leq 2$ (cf. Theorems 11 and 15); in the complex case we derive a result of McCullough $[12,13]$. Moreover, we obtain the classification of the 3-blocks, which was not known in the complex case (cf. Corollary 14).

The minimum fill-in fill $(G)$ of a graph $G$ is the minimum number of edges that need to be added to $G$ in order to obtain a chordal graph; this parameter has been studied, in particular, in connection with the Gaussian elimination process for real symmetric positive definite matrices (cf. Rose [15]). The following inequalities relating the sparsity order and the minimum fill-in have been shown in [17], solving a conjecture of [7].

Proposition 3. $\operatorname{ord}_{\mathbb{R}}(G) \leq \operatorname{fill}(G)+1, \quad \operatorname{ord}_{\mathbb{C}}(G) \leq 2 \cdot \operatorname{fill}(G)+1$.

We will see in Section 2 that these inequalities follow as an easy application of a result (cf. Theorem 6) about the dimension of faces of the cone $\mathcal{P}_{G}$.

Note that the difference between the minimum fill-in and the sparsity order can be arbitrarily large. Indeed, if $G$ is the clique sum of two graphs $G_{1}$ and $G_{2}$, then $\operatorname{ord}_{\mathbb{F}}(G)=\max \left(\operatorname{ord}_{\mathbb{F}}\left(G_{1}\right), \operatorname{ord}_{\mathbb{F}}(G)_{2}\right)$ while fill $(G)=\operatorname{fill}\left(G_{1}\right)+$ fill $\left(G_{2}\right)$. We will see in Section 3 examples of graphs (those in class $\mathcal{G}_{4}$ - they are not clique sums) having order 2 and an arbitrarily large minimum fillin. The complexity of computing the order of a graph is not known. On the other hand, evaluating the upper bound given by Proposition 3 is hard, since computing the minimum fill-in is NP-complete ([19]). 
Application to the completion problem. Let us now explain the link existing between the cone $\mathcal{P}_{G}$ and the completion problem for positive semidefinite matrices.

The matrix completion problem asks whether a given partial matrix can be completed so as to obtain a matrix satisfying a prescribed matrix property, in our case, being positive semidefinite. This problem has received a lot of attention in the literature; this is due, in particular, to its many applications, e.g., to statistics, molecular chemistry, distance geometry, etc. (Cf. the surveys by Johnson [9], Laurent [10] and further references there.)

Given a graph $G=(V, E)$ and a partial matrix $A=\left(a_{i j}\right)$ whose entries are specified only on the diagonal positions and on the off-diagonal positions corresponding to the edges of $G, A$ is said to be completable to a positive semidefinite matrix if there exist a positive semidefinite matrix $B$ such that $b_{i i}=a_{i i}(i \in V)$ and $b_{i j}=a_{i j}(i \neq j \in V$ and $i j \in E)$. Then, $\mathcal{C}_{G}$ denotes the subset of $\mathbb{R}^{V \cup E}$ consisting of all such completable partial matrices $A$. When $G$ is the complete graph, $\mathcal{C}_{G}$ is the cone of all positive semidefinite matrices, which is well known to be self polar. From this follows that the polar cone ${ }^{1}$ of $\mathcal{C}_{G}$ is equal to the projection on $\mathbb{R}^{V \cup E}$ of the cone $\mathcal{P}_{G}$.

Therefore, a partial matrix is completable to a positive semidefinite matrix if and only if its inner product with any extremal matrix in $\mathcal{P}_{G}$ is nonnegative. Hence, knowledge about the extremal matrices in $\mathcal{P}_{G}$ is useful for deciding completability of partial matrices. This fact motivates the study of extremal matrices in $\mathcal{P}_{G}$ and of the order of graph $G$. In view of the polarity relation between the cones $\mathcal{C}_{G}$ and $\mathcal{P}_{G}$, Theorem 1 is equivalent to the following result of [4] concerning the cone $\mathcal{C}_{G}$.

Theorem 4. A graph $G$ is chordal if and only if every partial matrix whose entries are specified on $G$ (and on the main diagonal) and for which all fully specified principal submatrices are positive semidefinite can be completed to a positive semidefinite matrix.

We now give some preliminaries about matrices and vector representations, leading to a reformulation for the sparsity order, and some notation on graphs.

Matrices and vector representations. An $n \times n$ matrix $X=\left(x_{i j}\right)$ with entries in $\mathbb{F}(=\mathbb{R}$ or $\mathbb{C})$ is Hermitian if $X^{*}=X$ and positive semidefinite

\footnotetext{
${ }^{1}$ Recall that the polar $C^{\circ}$ of a cone $C \subseteq \mathbb{F}^{d}$ is the set of all $y \in \mathbb{F}^{d}$ such that $y^{*} x \geq 0 \forall x \in C$. When the cone $C$ consists of $n \times n$ matrices, we view $C$ as a subset of $\mathbb{F}^{n^{2}}$ equipped with the usual inner product. That is, for two $n \times n$ matrices $A$ and $B$, their inner product $\langle A, B\rangle$ is defined as $\operatorname{Tr}\left(A^{*} B\right)=\sum_{i, j=1}^{n} a_{i j}^{*} b_{i j}$. Here, $z^{*}, a^{*}, A^{*}$ denote, respectively, the conjugate of $z \in \mathbb{F}$ (equal to $z$ if $\mathbb{F}=\mathbb{R}$ ), and the conjugate transpose of vector $a$ or matrix $A$.
} 
(then, we write: $X \succeq 0$ ) if $X$ is Hermitian and $x^{*} X x \geq 0$ for all $x \in \mathbb{F}^{n}$. Equivalently, $X \succeq 0$ if there exist vectors $u_{1}, \ldots, u_{n} \in \mathbb{F}^{k}(k \geq 1)$ such that $x_{i j}=u_{i}^{*} u_{j}$ for all $i, j=1, \ldots, n$; the sequence $\left(u_{1}, \ldots, u_{n}\right)$ is then called a Gram representation of $X$ and $X$ is called the Gram matrix of $u_{1}, \ldots, u_{n}$. Note that $\left\{u_{1}, \ldots, u_{n}\right\}$ and $X$ have the same rank. If $X$ has rank $k$, then it has a unique (up to orthogonal transformation) Gram representation in the $k$-dimensional space $\mathbb{F}^{k}$.

Let $G=(V, E)$ be a graph with $V=\{1, \ldots, n\}$ and let $X$ be an $n \times n$ positive semidefinite matrix with Gram representation $\left(u_{1}, \ldots, u_{n}\right)$. Then, $X \in \mathcal{P}_{G}$ if and only if the vectors $u_{1}, \ldots, u_{n}$ satisfy:

$$
u_{i}^{*} u_{j}=0 \text { for all } i j \in \bar{E},
$$

i.e., they form an orthogonal representation of $G$. Given a subset $A \subseteq E \cup \bar{E}$, set

$$
\begin{array}{ll}
U_{A}:=\left\{u_{i} u_{j}^{*}+u_{j} u_{i}^{*} \mid i j \in A\right\} & \text { if } \mathbb{F}=\mathbb{R}, \\
U_{A}:=\left\{u_{i} u_{j}^{*}, u_{j} u_{i}^{*} \mid i j \in A\right\} & \text { if } \mathbb{F}=\mathbb{C} .
\end{array}
$$

If $X \in \mathcal{P}_{G}$, then all matrices in $U_{\bar{E}}$ are orthogonal to the identity matrix. The following is shown in [1].

Proposition 5. Let $X \in \mathcal{P}_{G}$ with rank $k$ and Gram representation $\left(u_{1}, \ldots, u_{n}\right)$ in $\mathbb{F}^{k}$. Then, $X$ is extremal if and only if the identity matrix is (up to scalar multiple) the only $k \times k$ real symmetric matrix (resp. complex matrix) which is orthogonal to all matrices in $U_{\bar{E}}$ in the real case (resp. complex case). Equivalently, $X$ is extremal if and only if the following holds:

$$
\operatorname{rank}\left(U_{\bar{E}}\right)=\left(\begin{array}{c}
k+1 \\
2
\end{array}\right)-1(\mathbb{F}=\mathbb{R}), k^{2}-1(\mathbb{F}=\mathbb{C}) .
$$

Therefore,

(5) $\quad \operatorname{ord}_{\mathbb{F}}(G)=k \Longrightarrow|\bar{E}| \geq\left(\begin{array}{c}k+1 \\ 2\end{array}\right)-1(\mathbb{F}=\mathbb{R}), \frac{1}{2}\left(k^{2}-1\right)(\mathbb{F}=\mathbb{C})$.

A set of vectors $u_{1}, \ldots, u_{n} \in \mathbb{F}^{k}$ with rank $k$ and satisfying (2) and (4) is called a $k$-dimensional extremal orthogonal representation of $G$. Hence, the sparsity order of $G$ is equal to the largest $k$ for which there exists a $k$-dimensional extremal orthogonal representation of $G$. Proposition 5 turns out to be a direct consequence of the result from Theorem 6 about dimensions of faces of $\mathcal{P}_{G}$. 
Graphs. All graphs are assumed to be simple (i.e., without loops and parallel edges). Given a graph $G=(V, E)$ with $\bar{E}$ as set of non edges, $\bar{G}:=(V, \bar{E})$ is the complementary graph of $G$. As usual, $K_{n}$ denotes the complete graph on $n$ nodes, $K_{n, m}$ denotes the complete bipartite graph with colour classes of cardinalities $n$ and $m$, and $C_{n}$ denotes the circuit on $n$ nodes. Given a subset $U$ of $V, G[U]$ denotes the subgraph of $G$ induced by $U$, with node set $U$ and edge set $\{i j \in E \mid i, j \in U\}$. A subset $S \subseteq V$ is called a stable set of $G$ if $i j \notin E$ for all $i \neq j \in S$, and a clique if $i j \in E$ for all $i \neq j \in S$. The stability number $\alpha(G)$ is the maximum cardinality of a stable set in $G$. A subset $F \subseteq E$ is called a matching in $G$ if no two edges of $F$ have a common endnode.

Let $G_{1}=\left(V_{1}, E_{1}\right)$ and $G_{2}=\left(V_{2}, E_{2}\right)$ be two graphs such that the set $K:=V_{1} \cap V_{2}$ is a clique in both $G_{1}, G_{2}$ and there is no edge between a node of $V_{1} \backslash V_{2}$ and a node of $V_{2} \backslash V_{1}$. Then, the graph $G:=\left(V_{1} \cup V_{2}, E_{1} \cup E_{2}\right)$ is called the clique sum of the graphs $G_{1}$ and $G_{2}$ and the set $K$ is called a clique cutset of $G$.

A graph $G$ is said to be chordal (or triangulated) if it does not contain a circuit $C_{n}(n \geq 4)$ as an induced subgraph. Equivalently, $G$ is chordal if and only if $G$ is a clique sum of cliques (Dirac [3]).

Given an integer $m \geq 1$, let $G^{(m)}$ denote the graph obtained from $G$ by replacing every node $v \in V$ by a clique $K_{v}$ of cardinality $m$ and making any two nodes $i \in K_{u}, j \in K_{v}$ adjacent in $G^{(m)}$ if and only if the nodes $u$ and $v$ are adjacent in $G$.

\section{Relating the sparsity order of a graph and its deficiency in chordality}

In this section we show that the results from Propositions 3 and 5 follow as applications of a result concerning the structure of the faces of the cone $\mathcal{P}_{G}$.

Let $G=(V, E)$ be a graph with $V=\{1, \ldots, n\}$. A subset $F \subseteq \mathcal{P}_{G}$ is called a face of $\mathcal{P}_{G}$ if $X=Y+Z$ with $X \in F, Y, Z \in \mathcal{P}_{G}$ implies that $Y, Z \in F$. The extreme rays of $\mathcal{P}_{G}$ are its faces of dimension 1 . Given $X \in \mathcal{P}_{G}$, let $F_{\mathcal{P}_{G}}(X)$ denote the smallest (with respect to inclusion) face of $\mathcal{P}_{G}$ that contains $X$. Then,

$$
F_{\mathcal{P}_{G}}(X)=\left\{Y \in \mathcal{P}_{G} \mid \operatorname{Ker} X \subseteq \operatorname{Ker} Y\right\}
$$

(where $\operatorname{Ker} X=\left\{x \in \mathbb{R}^{n} \mid X x=0\right\}$ ); this relation was shown in [8] in the case when $G=K_{n}$ and the general case follows easily. Moreover, one can compute the dimension of the face $F_{\mathcal{P}_{G}}(X)$ in terms of parameters of $X$ (cf. Theorem 31.5 .3 in $[2])$. 
Theorem 6. Let $G=(V, E)$ be a graph, let $X \in \mathcal{P}_{G}$ with rank $k$ and Gram representation $\left(u_{1}, \ldots, u_{n}\right)$ in $\mathbb{F}^{k}$, and let $U_{\bar{E}}$ be defined by (3). Then,

(6) $\operatorname{dim} F_{\mathcal{P}_{G}}(X)=\left(\begin{array}{c}k+1 \\ 2\end{array}\right)-\operatorname{rank}_{\mathbb{R}}\left(U_{\bar{E}}\right)(\mathbb{F}=\mathbb{R}), k^{2}-\operatorname{rank}_{\mathbb{C}}\left(U_{\bar{E}}\right)(\mathbb{F}=\mathbb{C})$.

In particular, $X$ is extremal if and only if (4) holds.

Proof. Let $U$ denote the $k \times n$ matrix whose columns are the vectors $u_{1}, \ldots, u_{n}$. Then, $X=U^{*} U$. Call a $k \times k$ matrix $B$ a perturbation of $X$ if $X \pm \lambda B \in \mathcal{P}_{G}$ for some $\lambda>0$. Then, $\operatorname{dim} F_{\mathcal{P}_{G}}(X)$ is equal to the dimension of the set of perturbations of $X$. One can verify that $B$ is a perturbation of $X$ if and only if $B=U^{*} R U$ for some $k \times k$ Hermitian matrix $R$ satisfying

$$
\left\langle R, u_{i} u_{j}^{*}\right\rangle=\left\langle R, u_{j} u_{i}^{*}\right\rangle=0 \text { for all } i j \in \bar{E} .
$$

Let $\mathcal{U}$ denote the subspace of $\mathbb{F}^{k^{2}}$ (the set of $k \times k$ matrices) spanned by $U_{\bar{E}}$. In the real case, $\operatorname{dim} F_{\mathcal{P}_{G}}(X)$ is equal to the dimension of the orthogonal complement of $\mathcal{U}$ in the space $\mathcal{S}_{k}$ of real symmetric matrices and, thus, to $\left(\begin{array}{c}k+1 \\ 2\end{array}\right)$-rank $\mathrm{k}_{\mathbb{R}}\left(U_{\bar{E}}\right)$. (We have used here the fact that, for a symmetric matrix $B,\left\langle B, u_{i} u_{j}^{*}\right\rangle=0 \Longleftrightarrow\left\langle B, u_{i} u_{j}^{*}+u_{j} u_{i}^{*}\right\rangle=0$.) In the complex case, $\operatorname{dim} F_{\mathcal{P}_{G}}(X)$ is equal to the dimension of the set $\mathcal{H}_{k} \cap \mathcal{U}^{\perp}$, the orthogonal complement of $\mathcal{U}$ in the space $\mathcal{H}_{k}$ of $k \times k$ complex Hermitian matrices. However, the set $\mathcal{H}_{k} \cap \mathcal{U}^{\perp}$ has the same dimension as its superset $\mathbb{C}^{k^{2}} \cap \mathcal{U}^{\perp}$, which implies that $\operatorname{dim} F_{\mathcal{P}_{G}}(X)=k^{2}-\operatorname{rank}_{\mathbb{C}}\left(U_{\bar{E}}\right)$. Indeed, suppose that $\left\{R_{1}, \ldots, R_{p}\right\}$ is a set of linearly independent matrices in $\mathbb{C}^{k^{2}} \cap \mathcal{U}^{\perp}$. Note that for $R \in \mathbb{C}^{k^{2}} \cap \mathcal{U}^{\perp}$, both matrices $R+R^{*}$ and $i\left(R-R^{*}\right)$ belong to $\mathcal{H}_{k} \cap \mathcal{U}^{\perp}$. Moreover, at least one of the two systems $\left\{R_{1}+R_{1}^{*}, R_{2}, \ldots, R_{p}\right\}$ and $\left\{i\left(R_{1}-R_{1}^{*}\right), R_{2}, \ldots, R_{p}\right\}$ is linearly independent. Therefore, we can iteratively construct from $\left\{R_{1}, \ldots, R_{p}\right\}$ a set of $p$ linearly independent matrices in $\mathcal{H}_{k} \cap \mathcal{U}^{\perp}$.

We now indicate how Proposition 3 can be derived from Theorem 6 .

Let $G=(V, E)$ be a graph and set $k:=\operatorname{ord}_{\mathbb{F}}(G)$ and $p:=\operatorname{fill}(G)$. There exists a subset $F$ of $\bar{E}$ of cardinality $p$ such that the graph $H:=(V, E \cup F)$ is chordal. Let $X$ be an extremal matrix in $\mathcal{P}_{G}$ of rank $k$ and with Gram representation $\left(u_{1}, \ldots, u_{n}\right)$ in $\mathbb{F}^{k}$; thus, $X \in \mathcal{P}_{H}$. Set $\rho_{\mathbb{R}}:=\left(\begin{array}{c}k+1 \\ 2\end{array}\right)$ and $\rho_{\mathbb{C}}:=k^{2}$. By relation (6), we have:

$$
\begin{gathered}
\operatorname{rank}_{\mathbb{F}}\left(U_{\bar{E}}\right)=\rho_{\mathbb{F}}-\operatorname{dim} F_{\mathcal{P}_{G}}(X)=\rho_{\mathbb{F}}-1, \\
\operatorname{rank}_{\mathbb{F}}\left(U_{\bar{E} \backslash F}\right)=\rho_{\mathbb{F}}-\operatorname{dim} F_{\mathcal{P}_{H}}(X) .
\end{gathered}
$$

On the other hand,

$$
\operatorname{rank}_{\mathbb{F}}\left(U_{\bar{E}}\right) \leq \operatorname{rank}_{\mathbb{F}}\left(U_{\bar{E} \backslash F}\right)+\operatorname{rank}_{\mathbb{F}}\left(U_{F}\right) \leq \operatorname{rank}_{\mathbb{F}}\left(U_{\bar{E} \backslash F}\right)+\epsilon_{\mathbb{F}} \cdot|F|,
$$


setting $\epsilon_{\mathbb{R}}=1$ and $\epsilon_{\mathbb{C}}:=2$. This implies that

$$
\operatorname{dim} F_{\mathcal{P}_{H}}(X) \leq \epsilon_{\mathbb{F}} \cdot|F|+1=\epsilon_{\mathbb{F}} \cdot p+1
$$

There exist $d \leq \operatorname{dim} F_{\mathcal{P}_{H}}(X)$ extremal matrices $X_{1}, \ldots, X_{d} \in \mathcal{P}_{H}$ such that $X=X_{1}+\ldots+X_{d}$. This implies that $\operatorname{rank} X \leq \operatorname{rank} X_{1}+\ldots+\operatorname{rank} X_{d}$. Each matrix $X_{i}$ has rank 1 since $H$ is chordal. Therefore, $\operatorname{rank} X \leq d$ which, combined with the inequality: $d \leq \epsilon_{\mathbb{F}} \cdot p+1$, implies that $k=\operatorname{rank} X \leq \epsilon_{\mathbb{F}} \cdot p+1$. That is, $\operatorname{ord}_{\mathbb{F}}(G) \leq \epsilon_{\mathbb{F}} \cdot \operatorname{fill}(G)+1$.

\section{Graphs with sparsity order 2: The real case}

In this section, we characterize the graphs having sparsity order $\leq 2$ in the real case. The main result is Theorem 9 which gives two equivalent descriptions for these graphs; one is in terms of forbidden induced subgraphs and the other one shows how such graphs can be decomposed by means of clique sums using four basic classes of graphs.

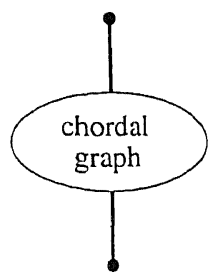

(1a) graph G

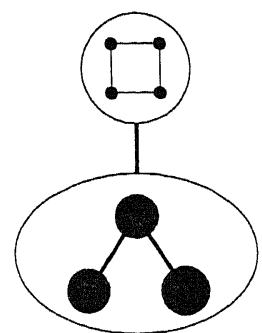

(2a) graph $\mathrm{G}$

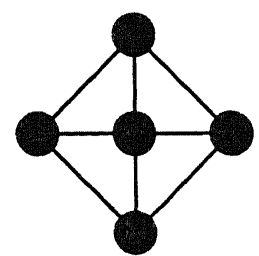

(4a) graph G

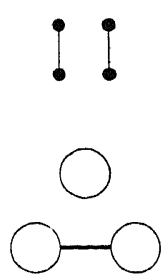

(2b) graph $\overline{\mathrm{G}}$

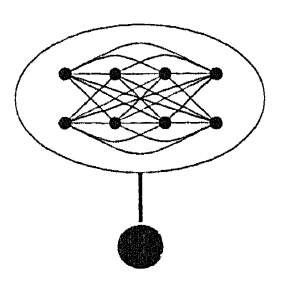

(3a) graph $\mathrm{G}$

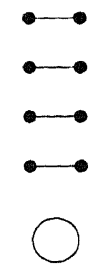

(3b) graph $\overline{\mathrm{G}}$

Fig. 1. Classes $\mathcal{G}_{1}, \mathcal{G}_{2}, \mathcal{G}_{3}$, and $\mathcal{G}_{4}$

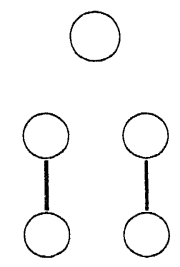

(4b) graph $\bar{G}$

We begin with introducing four classes of graphs having order $\leq 2$ over $\mathbb{R}$. These graphs are shown in Figure 1 .. For $i=1,2,3,4$, let $\mathcal{G}_{i}$ denote the class consisting of the graphs having the form shown in Figure 1. (ia) and 
of their induced subgraphs. We use the following convention in Figure 1.: A small dark dot indicates a node, a big dark sphere indicates a clique, while a big white sphere indicates a stable set; edges are indicated by lines, while a thick line between two spheres or between two sets of nodes shows that every node in one set is adjacent to every node in the other set.

For each class $\mathcal{G}_{i}$ with $i=2,3,4$, we picture not only graph $G \in \mathcal{G}_{i}$ but also its complementary graph $\bar{G}$, because the latter graph has a very simple form which will be used in the proof of Proposition 7. Note that a graph $G \in \mathcal{G}_{1}$ is obtained by adding two non adjacent nodes to a chordal graph $H$ and making them adjacent to all nodes of $H$ (and taking an induced subgraph of the resulting graph).

Remark that a graph in class $\mathcal{G}_{i}$ has minimum fill-in at most $i$ for $i=1,2,3$ while graphs in $\mathcal{G}_{4}$ may have an arbitrary large minimum fill-in.

Proposition 7. If $G \in \mathcal{G}_{1} \cup \mathcal{G}_{2} \cup \mathcal{G}_{3} \cup \mathcal{G}_{4}$, then $\operatorname{ord}_{\mathbb{R}}(G) \leq 2$.

Proof. If $G \in \mathcal{G}_{1}$, then $\operatorname{ord}_{\mathbb{R}}(G) \leq 2$ follows from Proposition 3 , since fill $(G) \leq$ 1.

Let $G \in \mathcal{G}_{i}$ for $i=2,3,4$. Let $X$ be an extremal matrix in the cone $\mathcal{P}_{G}$ having $\operatorname{rank} k:=\operatorname{ord}_{\mathbb{R}}(G)$ and with Gram representation $\left(u_{1}, \ldots, u_{n}\right)$ in $\mathbb{R}^{k}$. Then, by Theorem 6 ,

$$
\operatorname{rank}\left(U_{\bar{E}}\right)=\frac{1}{2}\left(k^{2}+k-2\right)
$$

We compute in each case the rank of the set $U_{\bar{E}}$. Consider first the case when $G \in \mathcal{G}_{2}$. Let $A, B$ denote the node sets corresponding to the two stable sets that are connected in $\bar{G}$ (cf. Figure 1 . (2b)) and set $a:=\operatorname{rank}\left\{u_{i} \mid i \in A\right\}$, $b:=\operatorname{rank}\left\{u_{i} \mid i \in B\right\}$. Then,

$$
\operatorname{rank}\left(U_{\bar{E}}\right) \leq 2+a b .
$$

We have that $a+b \leq k$ since every $u_{i}(i \in A)$ is orthogonal to every $u_{j}(j \in B)$; this implies that $a \bar{b} \leq \frac{1}{4} k^{2}$. Therefore, we have:

$$
\frac{1}{2}\left(k^{2}+k-2\right) \leq 2+\frac{1}{4} k^{2}
$$

from which it follows that $k \leq 2$. If $G \in \mathcal{G}_{3}$, then we have:

$$
\frac{1}{2}\left(k^{2}+k-2\right) \leq 4
$$

implying again that $k \leq 2$. Finally, if $G \in \mathcal{G}_{4}$, then we obtain in the same way as above that:

$$
\frac{1}{2}\left(k^{2}+k-2\right) \leq \frac{1}{4} k^{2}+\frac{1}{4} k^{2},
$$

which also implies that $k \leq 2$. 
We now characterize the graphs having order $\leq 2$ over $\mathbb{R}$. relies essentially on a graph-theoretic result concerning the chara in terms of forbidden induced subgraphs of the graphs in the $(i=1,2,3,4)$ and their clique sums. We first formulate this grap result whose proof, in view of its length, is delayed till Section 5

Theorem 8. The following assertions are equivalent for a grap.

(i) $G$ does not contain as an induced subgraph a circuit $C_{n}(n \geq$ of the graphs $A_{2}-A_{10}$ and $B_{1}-B_{6}$ (cf. Figure 2.).

(ii) $G$ is a clique sum of a set of graphs belonging to $\bigcup_{i=1}^{4} \mathcal{G}_{i}$ (cf. :

We show in Figure 2. the complementary graphs of a list of six $A_{1}-A_{10}, B_{1}-B_{6}$. Note that $A_{1}, B_{2}, A_{2}, B_{4}, B_{5}$ are, respectively $C_{5}$, the complete bipartite graph $K_{3,3}, K_{3,3}+e$ (add an edge to $K$ (delete an edge from $K_{3,3}$ ), $K_{3,3}+e \backslash f$.

Theorem 9. The following assertions are equivalent for a graf

(i) $\operatorname{ord}_{\mathbb{R}}(G) \leq 2$.

(ii) $G$ does not contain as an induced subgraph a circuit $C_{n}(n$ : of the graphs $A_{2}-A_{10}$ and $B_{1}-B_{6}$ (cf. Figure 2.).

(iii) $G$ is a clique sum of a set of graphs belonging to $\bigcup_{i=1}^{4} \mathcal{G}_{i}$ (cf.

Proof. The implication (i) $\Longrightarrow$ (ii) follows from the fact that th $(n \geq 5), A_{1}-A_{10}, B_{1}-B_{6}$ all have order $\geq 3$ (for this, it suffices t each of them a 3 -dimensional extremal orthogonal representat The implication (ii) $\Longrightarrow$ (iii) holds by Theorem 8 , while (iii) $=$ from relation (1) and Proposition 7.

The result from Theorem 9 can be seen as an analogue and ge of the corresponding characterization for graphs of order 1 , whic the following assertions are equivalent for a graph $G$ : (i) $\operatorname{ord}_{\mathbb{R}}$ $G$ is chordal; (iii) $G$ can be decomposed as a clique sum of cliq mention some applications of Theorem 9. The first application can test in polynomial time whether a given graph $G$ has order reals. Indeed, it suffices for this to first (i) decompose $G$ into gri clique cutsets by means of clique sums and then to (ii) test wh graphs produced by step (i) belong to $\bigcup_{i=1}^{4} \mathcal{G}_{i}$. Step (i) can be ] 

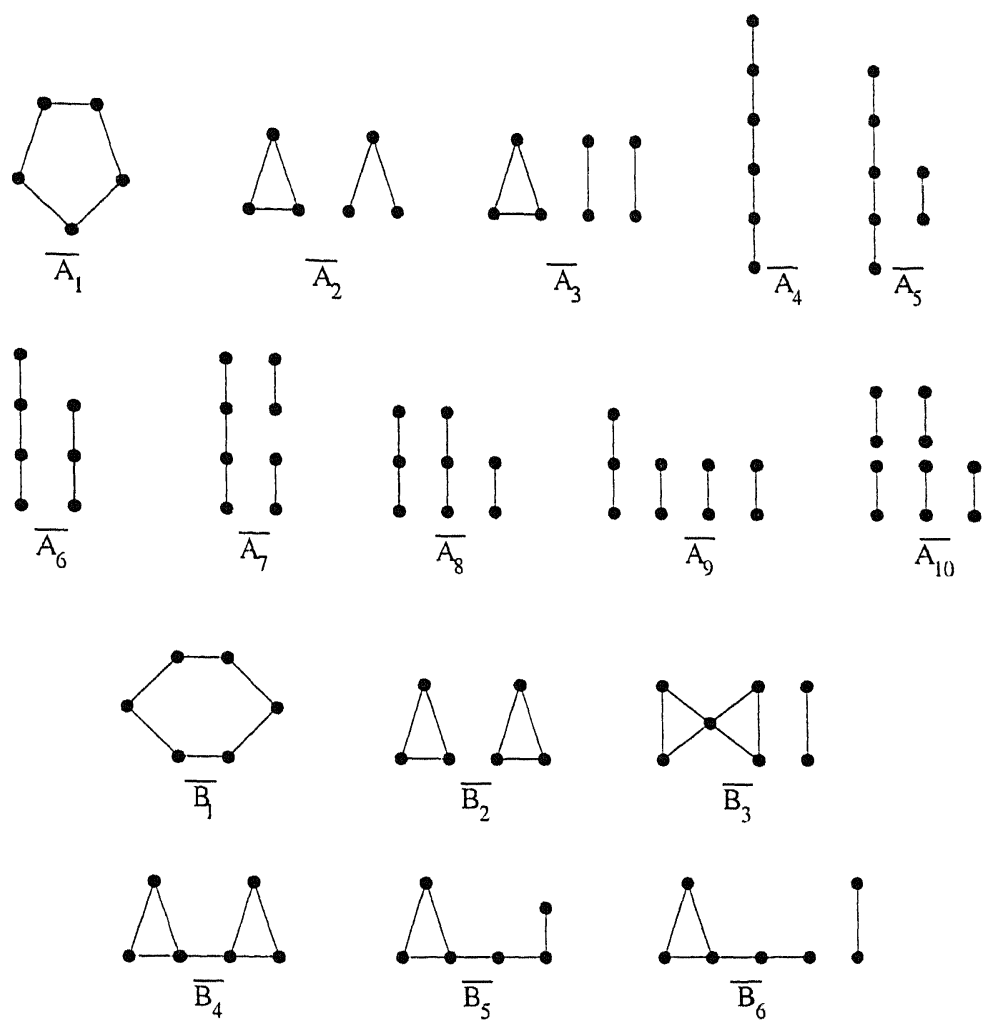

Fig. 2. Complements of graphs $A_{1}-A_{10}, B_{1}-B_{6}$ (the 3 -blocks over $\mathbb{R}$ )

time $O(n m)$ if $G$ has $n$ nodes and $m$ edges [18] and step (ii) can obviously be executed in polynomial time.

The second application is the classification of the 3-blocks over the reals, which was obtained by Agler et al. [1]. The only fact from [1] that we have used concerning the graphs $A_{1}-A_{10}, B_{1}-B_{6}$ is that they have order $\geq 3$ (which easily implies that they are 3 -blocks). But, we obtain 'for free' the hard part, which consists of showing that $A_{1}-A_{10}, B_{1}-B_{6}$ are the only 3-blocks.

Theorem 10. In the real case, the 3 -blocks are the graphs $A_{1}-A_{10}$ and $B_{1}-B_{6}$.

Proof. If $G$ is a 3-block then, by Theorem $9, G$ must contain one of the graphs $A_{1}-A_{10}, B_{1}-B_{6}$ as an induced subgraph and, thus, $G$ is equal to it (by the definition of a block). 
The third application of Theorem 9 is the characterization of the graphs $G$ whose powers $G^{(m)}$ all have order $\leq 2$ over $\mathbb{R}$.

Theorem 11. The following assertions are equivalent for a graph $G$.

(o) $\operatorname{ord}_{\mathbb{R}}\left(G^{(m)}\right) \leq 2$ for every integer $m \geq 1$.

(i) $\operatorname{ord}_{\mathbb{R}}\left(G^{(2)}\right) \leq 2$.

(ii) $G$ does not contain as an induced subgraph a circuit $C_{n}(n \geq 5)$ nor any of the graphs $A_{4}, B_{1}, D_{1}, D_{2}, D_{3}$ (cf. Figures 2. and 3.).

(iii) $G$ is a clique sum of a set of graphs belonging to the class $\mathcal{G}_{4}$ (cf. Figure 1.).

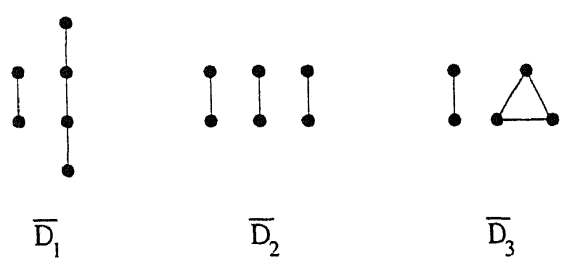

Fig. 3. Complements of graphs $D_{1}, D_{2}$, and $D_{3}$

The next lemma will be used in the proof of Theorem 11 and later as well.

\section{Lemma 12.}

(i) Let $H$ be a chordal graph that does not contain an induced path of length 3 and with stability number $\alpha(H)=2$. Then, its node set can be partitioned into $V_{0} \cup V_{1} \cup V_{2}$ in such a way that $V_{0} \cup V_{1}$ and $V_{0} \cup V_{2}$ are cliques and there is no edge between $V_{1}$ and $V_{2}$.

(ii) If $G \in \mathcal{G}_{1}$ contains neither $D_{1}$ nor $D_{3}$ as an induced subgraph, then $G \in \mathcal{G}_{4}$.

Proof. (i) As $H$ is chordal and is not a clique, there exists a clique cutset $K$ in $H$. Hence, the node set $V_{H}$ of $H$ can be partitioned into $N_{1} \cup N_{2} \cup K$, in such a way that there is no edge between $N_{1}$ and $N_{2}$. Moreover, both $N_{1}, N_{2}$ are cliques (since $\alpha(H)=2$ ). For $a=1,2$, set

$$
K_{a}:=\left\{k \in K \mid i k \notin E \text { for some } i \in N_{a}\right\} \text {. }
$$

Then, $K_{1} \neq \emptyset \Longrightarrow K_{2}=\emptyset$, a node $k \in K_{1}$ is not adjacent to any node of $N_{1}$ (else, one would find a path of length 3 in $H$ ), and $k \in K_{1}$ is adjacent to all 
nodes in $N_{2}$ (since $\alpha(H)=2$ ). Therefore, we can assume that $K_{2}=\emptyset$ and, then,

$$
V_{H}=N_{1} \cup\left(K \backslash K_{1}\right) \cup\left(K_{1} \cup N_{2}\right),
$$

where the sets $N_{1} \cup\left(K \backslash K_{1}\right)$ and $\left(K \backslash K_{1}\right) \cup K_{1} \cup N_{2}$ are cliques and there is no edge between the sets $N_{1}$ and $K_{1} \cup N_{2}$. Thus, (i) holds (setting $V_{0}:=K \backslash K_{1}$, $V_{1}:=N_{1}, V_{2}:=K_{1} \cup N_{2}$ ).

(ii) Let $G \in \mathcal{G}_{1}$ and let $H$ denote the chordal part in $G$ (cf. Figure 1. (1a)). If $G$ contains neither $D_{1}$ nor $D_{3}$ as an induced subgraph, then $H$ does not contain an induced path of length 3 and $\alpha(H) \leq 2$. We may assume that $H$ is not a clique (else we are done). Using (i) we conclude that $G$ has indeed the form of a graph in $\mathcal{G}_{4}$.

Proof of Theorem 11. (o) $\Longrightarrow(\mathrm{i})$ and (iii) $\Longrightarrow(0)$ are obvious. The implication (i) $\Longrightarrow$ (ii) follows from the corresponding implication in Theorem 9 ; indeed, the graphs $H:=D_{1}, D_{2}, D_{3}$ are forbidden as induced subgraphs of $G$ since $H^{(2)}$ contains $A_{6}, A_{8}, A_{2}$, respectively. Similarly, (ii) $\Longrightarrow$ (iii) follows from the corresponding implication in Theorem 9. Indeed, each of the graphs $A_{1}-A_{10}, B_{1}-B_{6}$ contains one of $A_{4}, B_{1}, D_{1}, D_{2}, D_{3}$ as an induced subgraph. Hence, under assumption (ii), we know that $G$ is a clique sum of a family of graphs belonging to $\bigcup_{i=1}^{4} \mathcal{G}_{i}$. In order to conclude the proof, it suffices now to verify that a graph $G \in \bigcup_{i=1}^{3} \mathcal{G}_{i}$ not containing $D_{1}, D_{2}, D_{3}$ necessarily belongs to $\mathcal{G}_{4}$. This is obvious for the classes $\mathcal{G}_{2}$ and $\mathcal{G}_{3}$ and Lemma 12 (ii) settles the case when $G \in \mathcal{G}_{1}$.

\section{Graphs with sparsity order 2: The complex case}

In this section we characterize the graphs having sparsity order $\leq 2$ over $\mathbb{C}$. As in the real case, we begin with exhibiting some basic classes of graphs having order $\leq 2$ over $\mathbb{C}$ as well as some examples of graphs with order $\geq 3$. Let $\mathcal{G}_{5}$ denote the class consisting of the graphs that can be obtained from a complete graph by deleting a matching of cardinality at most 3 . It follows from (5) that ord $\mathbb{C}(G) \leq 2$ if $G \in \mathcal{G}_{5}$ and McCullough (Prop. 2.6, [12]) has shown that $\operatorname{ord}_{\mathbb{C}}(G) \leq 2$ if $G \in \mathcal{G}_{4}$.

It is shown in [12] that ord $\operatorname{ord}_{\mathbb{C}}(G) \geq 3$ if $G$ is one of the graphs $C_{n}(n \geq 5), A_{4}$, $B_{1}$ (cf. Figure 2.), $D_{1}, D_{3}$ (cf. Figure 3.). We observe that the graphs $D_{4}$ and $D_{5}$ whose complements are shown in Figure 4 . also have order $\geq 3$. (The vector assignments indicated there provide a 3-dimensional extremal orthogonal representation.) In fact, the graphs $A_{4}, B_{1}, D_{1}, D_{3}, D_{4}, D_{5}$ all have order equal to 3 (by (5)). Therefore, the graphs $C_{n}(n \geq 5), A_{4}, B_{1}, D_{1}, D_{3}, D_{4}, D_{5}$ 


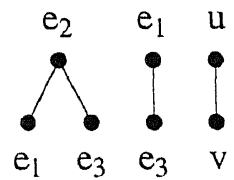

$\overline{\mathrm{D}_{4}}$

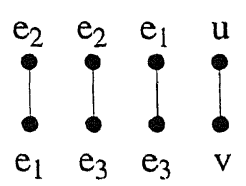

$\overline{D_{5}}$

Fig. 4. Complements of graphs $D_{4}$ and $D_{5}$

are forbidden induced subgraphs for the class of graphs having order $\leq 2$ over $\mathbb{C}$. As we now see, there are no other minimal forbidden induced subgraphs. The proof of this result relies again on a decomposition result, which follows quite easily from Theorem 8 .

Theorem 13. The following assertions are equivalent for a graph $G$.

(i) $\operatorname{ord}_{\mathbb{C}}(G) \leq 2$.

(ii) $G$ does not contain as an induced subgraph any of the graphs $C_{n}(n \geq 5)$, $A_{4}, B_{1}, D_{1}, D_{3}, D_{4}$, and $D_{5}$.

(iii) $G$ is a clique sum of a set of graphs belonging to $\mathcal{G}_{4} \cup \mathcal{G}_{5}$.

Proof. The implications (i) $\Longrightarrow$ (ii) and (iii) $\Longrightarrow$ (i) are clear. We now verify the implication (ii) $\Longrightarrow$ (iii). For this, let $G$ be a graph satisfying Theorem 13 (ii). Then, $G$ satisfies the condition (ii) from Theorem 9 (since the graphs $A_{2}, A_{3}, B_{2}-B_{6}$ all contain $D_{3}$; while $A_{5}, A_{6}, A_{7}$ contain $D_{1} ; A_{8}, A_{9}$ contain $D_{4}$; and finally $A_{10}$ contains $D_{5}$ ). Therefore, by Theorem $9, G$ is a clique sum of a set of graphs belonging to $\bigcup_{i=1}^{4} \mathcal{G}_{i}$. In order to conclude the proof, it suffices to verify that a graph $G \in \mathcal{G}_{1} \cup \mathcal{G}_{2} \cup \mathcal{G}_{3}$ satisfying Theorem 13 (ii) belongs, in fact, to $\mathcal{G}_{5}$. This is easy to see when $G \in \mathcal{G}_{2} \cup \mathcal{G}_{3}$ and Lemma 12 (ii) settles the case when $G \in \mathcal{G}_{1}$.

Therefore, the graphs having order $\leq 2$ over $\mathbb{C}$ can be recognized in polynomial time. Another application of Theorem 13 is the classification of the 3-blocks over $\mathbb{C}$.

Corollary 14. The 3-blocks over the field $\mathbb{C}$ are the graphs $C_{5}, B_{1}, A_{4}, D_{1}$, $D_{3}, D_{4}$, and $D_{5}$.

As last application of Theorem 13, we obtain the following result of McCullough $[12,13]$ characterizing the graphs $G$ whose powers $G^{(m)}$ all have orcler $\leq 2$ over $\mathbb{C}$. It turns out that we find the same graphs as in the real case. 
Theorem 15. The following assertions are equivalent for a graph $G$.

(o) $\operatorname{ord}_{\mathbb{C}}\left(G^{(m)}\right) \leq 2$ for every integer $m \geq 1$.

(i) $\operatorname{ord}_{\mathbb{C}}\left(G^{(2)}\right) \leq 2$.

(ii) $G$ does not contain as an induced subgraph a circuit $C_{n}(n \geq 5)$ nor any of the graphs $A_{4}, B_{1}, D_{1}, D_{2}, D_{3}$ (cf. Figures 2. and 3.).

(iii) $G$ is a clique sum of a set of graphs belonging to the class $\mathcal{G}_{4}$ (cf. Figure 1.).

Proof. The implications (o) $\Longrightarrow$ (i) and (iii) $\Longrightarrow(0)$ are clear. The implication (i) $\Longrightarrow$ (ii) follows from Theorem 13 and the fact that $D_{2}^{(2)}$ contains $D_{4}$. We now verify the implication (ii) $\Longrightarrow$ (iii). For this, let $G$ satisfy Theorem 15 (ii); then, $G$ satisfies Theorem 13 (ii) and, thus, is a clique sum of a set of graphs belonging to $\mathcal{G}_{4} \cup \mathcal{G}_{5}$. It suffices now to note that a graph belonging to $\mathcal{G}_{5}$ and satisfying Theorem 15 (ii) belongs, in fact, to $\mathcal{G}_{4}$.

McCullough $[12,13]$ has given an additional equivalent property for the graphs satisfying Theorem 15 (ii) (called by him 2-chordal), in terms of existence of a certain linear ordering of the nodes involving the notion of 'simplicial pair of nodes'; this is in analogy with the existence a perfect elimination ordering for chordal graphs, that involve the notion of simplicial node. Note that the 'hard' part in his proof lies also in proving the decomposition result via clique sums (the original proof given in [12] for this decomposition result was not correct; it was later corrected in [13]).

\section{Proof of Theorem 8}

This section is devoted to the proof of Theorem 8. The implication (ii) $\Longrightarrow$ (i) follows from the fact that the graphs $C_{n}(n \geq 5), A_{2}-A_{10}, B_{1}-B_{6}$ have no clique cutset and that they cannot occur as an induced subgraph of a graph in $\mathcal{G}_{i}(i=1,2,3,4)$.

We now turn to the proof of the reverse implication: $(i) \Longrightarrow$ (ii). The starting point of our proof was inspired by the proof given in [16] for the following result of Dirac [3]: Every chordal graph $G$ which is not a clique has a clique cutset. The latter result can be shown in the following manner.

Let $G$ be a chordal graph which is not a clique. Then, there exists a node $u$ which is not adjacent to all nodes in $V$. Let $S \subseteq V$ be a maximal subset of $V$ containing $u$ such that $G[S]$ is connected and the set

$$
N:=\{i \in V \backslash S \mid i \text { is adjacent to some node in } S\}
$$


is strictly contained in $V \backslash S$. Setting $\bar{N}:=V \backslash(S \cup N)$, we have partitioned $V$ into

$$
V=S \cup N \cup \bar{N}
$$

where $G[S]$ is connected, $\bar{N} \neq \emptyset$, and there is no edge between the sets $\bar{N}$ and $S$. It follows from the maximality assumption on $S$ that

$$
\text { every node of } N \text { is adjacent to every node of } \bar{N} \text {. }
$$

Moreover, $N$ is a clique and, thus, a clique cutset in $G$ (cf. the proof of Claim 3).

We now return to the proof of Theorem 8 (i) $\Longrightarrow$ (ii). For this, we let $G$ be a graph satisfying condition (i) (i.e., $G$ does not contain $C_{n}(n \geq 5)$, $A_{2}-A_{10}, B_{1}-B_{6}$ as an induced subgraph) and we assume that $G$ has no clique cutset. We show that $G$ belongs to one of the classes $\mathcal{G}_{i}, i=1,2,3,4$. We can assume that $G$ is not a clique (else, we are done). In the same manner as above, one can partition the node set $V$ into

$$
V=S \cup N \cup \bar{N}
$$

where $S \neq \emptyset, G[S]$ is connected, $\bar{N} \neq \emptyset$, there is no edge between the sets $\bar{N}$ and $S, N$ is not a clique, and relation (1) holds. The rest of the proof consists in a detailed analysis of the structure of the sets $S, N$ and $\bar{N}$, so that one can finally reach the conclusion that $G$ has indeed the form of a graph in $\bigcup_{i=1}^{4} \mathcal{G}_{i}$.

\subsection{Preliminary results and sketch of proof}

We group here a number of preliminary results on the structure of $G$ which will lead to several distinct cases that we have to consider. In what follows, we let $\bar{n}$ denote a given element of $\bar{N}$. For $s \in S$, we set

$$
N(s):=\{i \in N \mid \text { is } \in E\} .
$$

Claim 2. If st is an edge in $S$ such that $N(s) \cup N(t)$ is not a clique, then $N(s) \subseteq N(t)$ or $N(t) \subseteq N(s)$.

Proof. Assume that $s t$ is an edge in $S$ and that $N(s) \backslash N(t), N(t) \backslash N(s)$ are both non empty; we show that $N(s) \cup N(t)$ is a clique. For this, let $i \in N(s) \backslash N(t)$ and $j \in N(t) \backslash N(s)$; then, $i j \in E$ (else, $(\bar{n}, i, s, t, j$ ) would be an induced $C_{5}$ ). Let $i^{\prime}$ be another node in $N(s) \backslash N(t)$; then, $i i^{\prime} \in E$ (else, we find 
$B_{5}$ on $\left\{s, t, i, i^{\prime}, j, \bar{n}\right\}$ ). Let $k \in N(s) \cap N(t)$; then, $k i \in E$ (else, we find $A_{4}$ or $B_{1}$ on $\{\bar{n}, s, t, i, j, k\}$ depending whether $k j \in E)$. Finally, if $k, k^{\prime} \in N(s) \cap N(t)$, then $k k^{\prime} \in E$ (else, we find $A_{5}$ on $\left\{s, t, k, k^{\prime}, i, j, \bar{n}\right\}$ ).

Claim 3. If $i, j \in N$ are two non adjacent nodes in $N$, then there exists $s \in S$ which is adjacent to both $i$ and $j$.

Proof. Let $i, j \in N$ be non adjacent and assume that no $s \in S$ is adjacent to both $i, j$. There exist $s, t \in S$ such that $s i, t j \in E$ and $s j, t i \notin E$. Consider a shortest path $P$ in $S$ from $s$ to $t$. Then, this path $P$ together with the edges si, $i \bar{n}, \bar{n} j, j t$ yields a circuit of length $\geq 5$ in $G$, a contradiction.

As a consequence of Claim 3 , we obtain that

$$
G[\bar{N}] \text { is chordal. }
$$

Indeed, suppose that $G[\bar{N}]$ contains an induced $C_{4}$; let $i, j \in N$ be nonadjacent, and let $s \in S$ be adjacent to $i$ and $j$. Then, we find $B_{3}$ on the nodes of $C_{4}$ and $i, j, s$, a contradiction.

Claim 5. Let $I \subseteq N$ be a stable set in $N$ of cardinality $|I| \geq 3$. Then, there exists a unique node $s \in S$ which is adjacent to all nodes in $I$.

Proof. We proceed by induction on $|I| \geq 3$. Suppose first that $|I|=3, I=$ $\{i, j, k\}$. If there exists no node in $S$ adjacent to $i, j, k$ then, by Claims 3 and 2 , there exist pairwise nonadjacent nodes $r, s, t \in S$ such that $r i, r j, s i, s k, t j, t k \in$ $E$ and $r k, s j, t i \notin E$; this gives an induced circuit $C_{6}$ in $G$, a contradiction. Now, if $s, t$ are two distinct nodes in $S$ adjacent to $i, j$ and $k$, then we find $A_{2}$ or $B_{2}$ on $\{i, j, k, s, t, \bar{n}\}$ depending whether $s, t$ are adjacent or not. Hence, the result holds when $|I|=3$. Suppose now that $I=\left\{i_{1}, \ldots, i_{p}\right\}$ with $p \geq 4$ and that no node of $S$ is adjacent to all elements of $I$. By the induction assumption, we may assume that, for every $j=1, \ldots, p$, there exists $s_{j} \in S$ adjacent to all nodes in $I \backslash\left\{i_{j}\right\}$; then, the subgraph of $G$ induced by nodes $\bar{n}, s_{1}, s_{p}, i_{p-2}, i_{p-1}, i_{p}$ is $B_{4}$. Hence, there exists $s \in S$ adjacent to all nodes in $I$; uniqueness follows from the case $|I|=3$.

Claim 6. Let $i, j, k \in N$ be distinct nodes such that $G[\{i, j, k\}]$ has exactly one edge. Then, there exists a node $s \in S$ which is adjacent to $i, j$, and $k$.

Proof. Suppose the claim does not hold. Say, $i j \in E$ and $i k, j k \notin E$. Then, there exist $s, t \in S$ such that $s$ is adjacent to $i, k$ but not to $j$ and $t$ is adjacent to $j, k$ but not to $i$. Then, st $\in E$ (else, we find $C_{5}$ on $s, t, i, j, k$ ) and we find $B_{1}$ on $\bar{n}, s, t, i, j, k$. 
Claim 7. Let $I, J \subseteq N$ be distinct maximal stable sets in $N$. If $I \cap J \neq \emptyset$, then any node $s \in S$ which is adjacent to all elements in $I$ is adjacent to all elements in $J$.

Proof. Suppose not. Let $s \in S$ be adjacent to all elements in $I$ and let $j \in J \backslash I$ such that $s j \notin E$. By maximality of $I$, there exists $i \in I$ such that $i j \in E$. Let $k \in I \cap J$ and let $t \in S$ be adjacent to $i, j$, and $k$ (which exists by Claim 6). Then, we find $B_{4}$ or $B_{5}$ on $\bar{n}, s, t, i, j, k$ (depending whether $s t \in E$ or not).

Claim 8. Let $(i, h, j, k)$ be an induced $C_{4}$ in $N$ (i.e., $i h, j h, i k, j k \in E, i j, h k \notin$ $E)$. Then, any node $s \in S$ which is adjacent to $i$ and $j$ is adjacent to $h$ and $k$. Moreover, every node $x \in N \backslash\{i, j, h, k\}$ is adjacent to at least three nodes in $\{i, j, h, k\}$.

Proof. Let $s \in S$ be adjacent to $i$ and $j$ and suppose that $s$ is not adjacent, say, to $k$. Let $t \in S$ be adjacent to $h$ and $k$. Suppose in a first step that sh $\notin E$. If $t$ is adjacent to both $i, j$, then we find $B_{3}$ or $B_{6}$ on the nodes $\bar{n}, s, t, i, j, h, k$ (depending whether $s, t$ are adjacent); if $t$ is adjacent to one of $i, j$, then we find $A_{2}$ or $B_{5}$ and, if $t$ is not adjacent to $i, j$, then we find $B_{2}$ or $B_{4}$ on the nodes $s, t, i, j, h, k$. Therefore, we have that $s h \in E$ and, similarly, $t i \in E$. Then, we find $A_{5}$ or $B_{6}$ on $\{\bar{n}, s, t, i, j, h, k\}$ when $t j \in E$ and we find $A_{4}$ when $t j \notin E$ (on $\{s, t, i, j, h, k\}$ if $s t \notin E$ and on $\{\bar{n}, s, t, i, j, k\}$ if $s t \in E$ ).

We now prove the second assertion of the claim. For this, consider $x \in$ $N \backslash\{i, j, h, k\}$ such that $x i \notin E$. Let $s \in S$ which is adjacent to $x, i, j$ (which exists by Claim 6); then $s$ is adjacent to $h$ and $k$. Hence, the subgraph of $G$ induced by $\{\bar{n}, s, x, i, j, h, h\}$ is $A_{3}, A_{5}, B_{3}$, or $B_{6}$ if one of the edges $x j, x h, x k$ is missing.

Corollary 9. If $\bar{N}$ is not a clique, then $G[N]$ is chordal and there are at least two edges among any three nodes in $N$.

Proof. Let $\bar{n}_{1}, \bar{n}_{2}$ be two non adjacent nodes in $\bar{N}$. Suppose first that $(i, h, j, k)$ is an induced $C_{4}$ in $N$. Let $s \in S$ be adjacent to $i, j, h, k$ (which exists by Claims 3 and 8 ); thus, we find $A_{3}$ on $\left\{\bar{n}_{1}, \bar{n}_{2}, s, i, j, h, k\right\}$. This shows that $G[N]$ is chordal. Suppose now that $i, j, k$ are distinct nodes in $N$ having at most one edge among them. Then, there exists $s \in S$ adjacent to $i, j, k$ (by Claims 5 and 6 ); thus, we find $B_{2}$ or $A_{2}$ on $\left\{\bar{n}_{1}, \bar{n}_{2}, s, i, j, k\right\}$.

Let $\nu$ denote the largest cardinality of an induced matching in $\bar{G}[N]$, the complementary graph of $G[N]$. Then, $\nu \geq 1$ since $N$ is not a clique and

$$
\nu \leq 3
$$


Indeed, suppose that $\nu \geq 4$ and let $\left\{i_{a} j_{a} \mid a=1,2,3,4\right\}$ be an induced matching in $\bar{G}[N]$. By Claims 3 and 8 , there exists a node $s \in S$ which is adjacent to all nodes $i_{a}, j_{a}, a=1, \ldots, 4$. Then, we find $A_{10}$ on $\left\{\bar{n}, s, i_{a}, j_{a}(a=1,2,3,4)\right\}$. Note that $\nu \geq 2$ if and only if $G[N]$ is not chordal.

We can now describe the overal structure of the proof. We will organize our discussion according to the value of the parameter $\nu=1,2,3$ (by (10)). In the case when $\nu=1$, i.e., when the graph $G[N]$ is chordal, it will be convenient to consider separately the two cases when $\alpha(G[N])=2$ and when $\alpha(G[N]) \geq 3$. To summarize, the proof will consist of examining the following disjoint cases:

Case A: $\nu=1$ and $\alpha(G[N])=2$; then, we show that $G \in \mathcal{G}_{1} \cup \mathcal{G}_{4}$.

Case B: $\nu=1$ and $\alpha(G[N]) \geq 3$; then, we show that $G \in \mathcal{G}_{1}$.

Case C: $\nu \in\{2,3\}$; then, we show that $G \in \mathcal{G}_{\nu}$.

\subsection{Case A}

We assume here that $G[N]$ is chordal with stability number $\alpha(G[N])=2$. Let $K \subseteq N$ be a clique cutset in $G[N]$. Then, $N$ can be partitioned as

$$
N=K \cup N_{1} \cup N_{2}
$$

where $N_{1}, N_{2} \neq \emptyset$ and there is no edge between the sets $N_{1}$ and $N_{2}$. Moreover, both $N_{1}$ and $N_{2}$ are cliques (since $\alpha(G[N])=2$ ). We show that $G$ belongs to $\mathcal{G}_{1} \cup \mathcal{G}_{4}$. For convenience, we introduce the following sets:

$$
\begin{aligned}
& S_{1}:=\left\{s \in S \mid s \text { is adjacent to } N_{1} \text { but not to } N_{2}\right\}, \\
& S_{2}:=\left\{s \in S \mid s \text { is adjacent to } N_{2} \text { but not to } N_{1}\right\},
\end{aligned}
$$

$S_{12}:=\left\{s \in S \mid s\right.$ is adjacent to $N_{1}$ and $\left.N_{2}\right\}, \quad S_{0}:=S \backslash\left(S_{1} \cup S_{2} \cup S_{12}\right)$ and, for $a=1,2$,

$$
K_{a}:=\left\{k \in K \mid i k \notin E \text { for some } i \in N_{a}\right\} \text { and } K_{0}:=K \backslash\left(K_{1} \cup K_{2}\right) .
$$

Given a set $A \subseteq V$ and $u \in V \backslash A$, we say that $u$ is adjacent to $A$ if $u$ is adjacent to some element in $A$. Moreover, a path connecting a node of $S_{1}$ to a node of $S_{2}$ whose set of internal nodes is contained in $S_{0}$ is called a path from $S_{1}$ to $S_{2}$ via $S_{0}$. We have:

$$
K_{1} \cap K_{2}=\emptyset ; N_{1} \cup K_{2} \text { and } N_{2} \cup K_{1} \text { are cliques, }
$$

since $\alpha(G[N])=2$. Moreover,

$$
S_{12} \neq \emptyset
$$


Indeed, given $i_{1} \in N_{1}, i_{2} \in N_{2}$, there exists (by Claim 3) a node $s \in S$ which is adjacent to $i_{1}$ and $i_{2}$; thus, $s \in S_{12}$. The following observation will be used repeatedly.

Claim 11. There does not exist an induced path from $S_{1}$ to $S_{2}$ via $S_{0}$. Moreover, any induced path contained in $S_{0} \cup S_{1} \cup S_{2}$ is contained in $S_{0} \cup S_{1}$ or in $S_{0} \cup S_{2}$.

Proof. Suppose that there exists an induced path $\left(s_{1}, u_{1}, \ldots, u_{p}, s_{2}\right)$ where $s_{1} \in S_{1}, s_{2} \in S_{2}$, and $u_{1}, \ldots, u_{p} \in S_{0}(p \geq 0)$. Let $i_{a} \in N_{a}$ be adjacent to $s_{a}$, for $a=1,2$. Then, $\left(i_{1}, s_{1}, u_{1}, \ldots, u_{p}, s_{2}, i_{2}, \bar{n}\right)$ is an induced circuit of length $\geq 5$ in $G$, yielding a contradiction. The second assertion in the claim follows easily.

By definition, every node of $S_{12}$ is adjacent to at least one node in $N_{1}$ and in $N_{2}$. More strongly, we have:

(12) Every node $s \in S_{12}$ is adjacent to every node in $N_{1} \cup N_{2} \cup K_{1} \cup K_{2}$.

This follows using Claim 7, since any two non adjacent nodes of $N$ form a maximal stable set in $G[N]$. Indeed, let $s \in S_{12}$ and let $i_{1} \in N_{1}, i_{2} \in N_{2}$ be adjacent to $s$. Then, $s$ is adjacent to every other node $j_{1} \in N_{1}$ since $\left\{i_{1}, i_{2}\right\}$ and $\left\{j_{1}, i_{2}\right\}$ are two intersecting maximal stable sets. Moreover, if $k \in K_{1}$ is not adjacent to some $j_{1} \in N_{1}$, then $s$ is adjacent to $k$ since $\left\{k, j_{1}\right\}$ is a maximal stable set meeting $\left\{j_{1}, i_{2}\right\}$.

Claim 13. The graph $G\left[S_{12} \cup K \cup \bar{N}\right]$ is chordal.

Proof. We already know that $G[K \cup \bar{N}]$ is chordal (using (4)); hence, a possible $C_{4}$ is necessarily contained in $K \cup S_{12}$ and has at least two nodes in $S_{12}$. Let $i_{1} \in N_{1}$ and $i_{2} \in N_{2}$. If $(i, j, s, t)$ is an induced $C_{4}$ with $i, j \in K$ and $s, t \in S_{12}$, then $i, j \in K_{0}$ (by (12)) and we find $A_{5}$ on $\bar{n}, s, t, i, j, i_{1}, i_{2}$. In the case when $(i, r, s, t)$ is an induced $C_{4}$ with $i \in K$ and $r, s, t \in S$, then $i \in K_{0}$ and we find $B_{6}$ on $\bar{n}, r, s, t, i, i_{1}, i_{2}$. Finally, if $(r, s, t, u)$ is an induced $C_{4}$ contained in $S_{12}$, then we find $B_{3}$ on $\bar{n}, r, s, t, u, i_{1}, i_{2}$.

Our next objective is to show that $S=S_{12}$, i.e., that the set $T:=S_{0} \cup S_{1} \cup S_{2}$ is empty. Given $s \in T$, set

$$
X_{s}:=\left\{x \in S_{12} \cup N \mid s x \in E\right\} .
$$

Claim 14. $X_{s}$ is a clique for every $s \in T$ and $X_{s} \cup X_{t}$ is a clique for every edge st in $T$. 
Proof. Note first that, if $x, y$ are two non adjacent nodes in $S_{12} \cup N$, then one of the following holds: either $x \in S_{12}, y \in S_{12} \cup K_{0}$; or $x \in K_{1}, y \in N_{1}$ (or the symmetric case: $x \in K_{2}, y \in N_{2}$ ); or $x \in N_{1}, y \in N_{2}$.

Suppose that $X_{s}$ is not a clique for some $s \in T$ and let $x, y$ be nonadjacent nodes in $X_{s}$. If $x \in S_{12}$, then $N(s) \subseteq N(x)$ (by Claim 2, since $N(x)$ is not a clique) and, thus, $y \notin N$ which, in view of the above observation, means that $y \in S_{12}$. But, then, we find $B_{4}$ or $B_{5}$ on $\left\{\bar{n}, s, x, y, i_{1}, i_{2}\right\}$ (where $i_{1} \in N_{1}$, $i_{2} \in N_{2}$ ). We cannot have: $x \in N_{1}, y \in N_{2}$; therefore, $x \in K_{1}, y \in N_{1}$ and, thus, $s \in S_{1}$. Then, $s$ must be adjacent to any $i_{2} \in N_{2}$ (by Claim 7 , since $\left\{y, i_{2}\right\}$ is a maximal stable set in $N$ meeting $\{x, y\}$ ), contradicting the fact that $s \in S_{1}$. Hence, $X_{s}$ is a clique.

Suppose now that $X_{s} \cup X_{t}$ is not a clique for some edge $s t$ in $T$. Then, there exist two nonadjacent nodes $x, y$ in $X_{s} \cup X_{t}$ with, say, $x \in X_{s} \backslash X_{t}$ and $y \in X_{t} \backslash X_{s}$. We can assume, e.g., that $s, t \in S_{0} \cup S_{1}$. Again we cannot have: $x \in N_{1}, y \in N_{2}$. Hence, given $i_{2} \in N_{2},\left(i_{2}, x, s, t, y\right)$ is an induced $C_{5}$ in the case when $x \in S_{12}$ and $y \in S_{12} \cup K_{0}$; in the case when $x \in K_{1}, y \in N_{1}$, then $(\bar{n}, x, s, t, y)$ is an induced $C_{5}$.

If $T \neq \emptyset$, we let $A$ denote a maximal subset of $T$ for which $G[A]$ is connected and the set $X(A):=\bigcup_{a \in A} X_{a}$ is a clique.

Claim 15. There is no edge between the sets $A$ and $T \backslash A$.

Proof. Assume that $a \in A$ is adjacent to $b \in T \backslash A$. By maximality of $A$, we deduce that $X(A) \cup X_{b}$ is not a clique; hence, there exist two nonadjacent nodes $x \in X(A)$ and $y \in X_{b}$. Let $a_{0} \in A$ be adjacent to $x$. As $x, y$ are not adjacent, we deduce from Claim 14 that $a_{0} \neq a, a_{0} b, a_{0} y, b x \notin E$. Let $\left(a_{0}, a_{1}, \ldots, a_{p}, a\right)$ be a shortest path connecting $a_{0}$ and $a$ in $A$ (possibly $p=0$ if $\left.a_{0} a \in E\right)$. Together with nodes $x, y$, this yields an induced path $P$ of length $\geq 4$ from $x$ to $y$ and whose internal nodes belong to $T$. Now, by Claim 11, we may assume that all internal nodes of $P$ belong to $S_{0} \cup S_{1}$. Therefore, the path $P$ together with edges $i_{2} x, i_{2} y$ (resp. with edges $\bar{n} x, \bar{n} y$ ) yields an induced circuit of length $\geq 6$ in $G$ when $x \in S_{12}$ and $y \in S_{12} \cup K_{0}$ (resp. when $x \in K_{1}$ and $y \in N_{1}$ ).

We can now deduce that

$$
T=S_{0} \cup S_{1} \cup S_{0}=\emptyset ; \text { that is, } S=S_{12} .
$$

For, if $T \neq \emptyset$, then $A \neq \emptyset$ and $X(A)$ is a clique cutset in $G$. (To see it, note that there is no edge between $A$ and the sets $T \backslash A,\left(S_{12} \cup N\right) \backslash X(A)$ and $\bar{N}$. Hence, if we delete the clique $X(A)$ in $G$, we obtain a graph in which $A$ is disconnected from the rest of the graph.) 
Corollary 16. If $\left|N_{1}\right|=\left|N_{2}\right|=1$ and $K_{1}=K_{2}=\emptyset$, then $G \in \mathcal{G}_{1}$.

Proof. Indeed, under this assumption, we have that $N_{1}=\left\{i_{1}\right\}, N_{2}=\left\{i_{2}\right\}$ where both $i_{1}$ and $i_{2}$ are adjacent to all nodes in $V \backslash\left\{i_{1}, i_{2}\right\}=S \cup K \cup \bar{N}$. As $G\left[V \backslash\left\{i_{1}, i_{2}\right\}\right]$ is chordal (by Claim 13), we obtain that $G \in \mathcal{G}_{1}$.

From now on, we can assume without loss of generality that the following holds:

$$
\left|N_{1}\right| \geq 2, \text { or } K_{1} \neq \emptyset
$$

Then, $\bar{N}$ is a clique by Corollary 9 . Moreover,

$S$ is a clique and every node of $S$ is adjacent to every node of $N$.

Indeed, it follows from assumption (17) that $\bar{G}[N]$ contains a path $(i, j, k)$ of length 2 (choosing, either $i, k \in N_{1}, j \in N_{2}$; or $i \in K_{1}, j \in N_{1}, k \in N_{2}$ where $i j \notin E)$. Therefore, if $s, t$ are two non adjacent nodes of $S$, then we find $A_{2}$ on the set $\{i, j, k, s, t, \bar{n}\}$. Hence, $S$ is a clique. Suppose now that $s \in S$ is not adjacent to some node $h \in N$; then, $h \in K_{0}$ by (12). Let $t \in S$ be adjacent to $h$. Then, we find $A_{6}$ on the set $\{\bar{n}, s, t, h, i, j, k\}$.

Hence, we know the following information about $G$ : The sets $S$ and $\bar{N}$ are cliques, every node of $S \cup \bar{N}$ is adjacent to every node of $N$, and $G[N]$ is chordal. This implies:

Corollary 18. If $|S|=|\bar{N}|=1$, then $G \in \mathcal{G}_{1}$.

Henceforth, we can now assume, moreover, that

$$
\max (|S|,|\bar{N}|) \geq 2 \text {. }
$$

This implies that

$G[N]$ does not contain an induced path of length 3.

Indeed, $\bar{G}[S \cup \bar{N}]$ contains a path of length 2 by (19); hence, if $G[N]$ would contain an induced path of length 3 , we would find graph $A_{6}$.

Therefore, by Lemma 12 (i), we know that $N$ can be partitioned into

$$
N=V_{1} \cup V_{0} \cup V_{2}
$$

where $V_{0} \cup V_{1}$ and $V_{0} \cup V_{2}$ are cliques and there is no edge between $V_{1}$ and $V_{2}$. (Namely, $V_{0}=K_{0}, V_{1}=N_{1}$ and $V_{2}=K_{1} \cup N_{2}$.) We can now conclude that $G$ belongs to class $\mathcal{G}_{4}$ (with the cliques $V_{1}, S, V_{2}, \bar{N}$ forming the outer circuit and $V_{0}$ as central clique). This concludes the proof in case $\mathrm{A}$. 


\subsection{Case B}

We assume here that $G[N]$ is chordal with stability number $\alpha(G[N]) \geq 3$. We show that $G \in \mathcal{G}_{1}$. For this, let $I$ be a maximal stable set in $N ;|I| \geq 3$. By Claim 5, there exists an element $s_{I} \in S$ which is adjacent to all nodes in I. A first easy observation is that

$$
|\bar{N}|=1 .
$$

Indeed, if $\bar{n}_{1}, \bar{n}_{2} \in \bar{N}$ and $i, j, k \in I$, then we find $A_{2}$ or $B_{2}$ on $\left\{\bar{n}_{1}, \bar{n}_{2}, s_{I}, i, j, k\right\}$. Next, we observe that

$s_{I}$ is adjacent to every node of $N$.

Indeed, let $h \in N \backslash I$. If $h i \notin E$ for some $i \in I$, then $s_{I}$ is adjacent to $h$ by Claim 7. If $h i \in E$ for all $i \in I$, then $s_{I} h \in E$ for, if not, we find $A_{2}$ on $\left\{\bar{n}, s_{I}, h, i, j, k\right\}$ (where $i, j, k \in I$ ). At this point, we can already conclude that

$$
\text { if } S=\left\{s_{I}\right\} \text {, then } G \in \mathcal{G}_{1} \text {. }
$$

Indeed, $s_{I}$ and $\bar{n}$ are both adjacent to all elements of $N$ and $G[N]$ is chordal. We now show that the set $T:=S \backslash\left\{s_{I}\right\}$ is empty. For this, note first that every node $s \in T$ is adjacent to at most one node of $I$.

Indeed, we know from Claim 5 that $s$ is adjacent to at most two nodes of $I$. If $s \in T$ is adjacent to $i, j \in I$ and if $k \in I \backslash\{i, j\}$, then we find $B_{4}$ or $B_{5}$ on $\left\{\bar{n}, s, s_{I}, i, j, k\right\}$. For $s \in T$, set

$$
Y_{s}:=\{x \in N \mid s x \in E\} .
$$

Claim 22. For every $s \in T, Y_{s}$ is a clique and, for every edge st in $T, Y_{s} \cup Y_{t}$ is a clique.

Proof. Suppose that $x, y \in Y_{s}$ are not adjacent where $s \in T$. Let $i, j \in I$ be botl non adjacent to $s$ ( $\operatorname{such} i, j$ exist by (21)). Then, we have that $x i, y i \in E$ (for, if not, we find $A_{4}, B_{4}$ or $B_{5}$ on $\left\{\bar{n}, s, s_{I}, x, y, i\right\}$ ). Similarly, $x j, y j \in E$. Hence, we have found $(x, i, y, j)$ as induced $C_{4}$ in $G[N]$, which contradicts our assumption that $G[N]$ is chordal.

Suppose now that $Y_{s} \cup Y_{t}$ is not a clique for some edge $s t$ in $T$; let $x \in Y_{s} \backslash Y_{t}$, $y \in Y_{l} \backslash Y_{s}$ be non adjacent. Then, $(\bar{n}, x, s, t, y)$ is an induced $C_{5}$.

Let $A$ be a maximal subset of $T$ such that $G[A]$ is connected and the set $Y(A):=\bigcup_{a \in A} Y_{a}$ is a clique. One can verify that

there is no edge between the sets $A$ and $T \backslash A$. 
(The proof is similar to that of Claim 15. Namely, if $a \in A$ is adjacent to $b \in T \backslash A$, then we find two non adjacent nodes $x \in Y(A), y \in Y_{b}$ and an induced path $P$ from $x$ to $y$ whose internal nodes belong to $T$. This path $P$ together with edges $\bar{n} x, \bar{n} y$ yields an induced circuit of length $\geq 6$.)

From this it follows that $A=\emptyset$ (otherwise, the clique $Y(A) \cup\left\{s_{I}\right\}$ would be a clique cutset in $G$ ). Therefore, $T=\emptyset$, which shows that $S=\left\{s_{I}\right\}$ and, thus, $G \in \mathcal{G}_{1}$ by (20). This concludes the proof in Case B.

\subsection{Case C}

We assume here that $\nu \in\{2,3\}$; thus, $G[N]$ is not chordal. By Corollary 9 , this implies that

$\bar{N}$ is a clique.

Let $\left\{i_{a} j_{a} \mid a=1, \ldots, \nu\right\}$ be an induced matching of maximum cardinality in $\bar{G}[N]$. In view of Claim 8, every node of $N$ is non adjacent to at most one of the $i_{a}, j_{a}$ 's. This leads us to defining the following sets:

$$
I_{a}:=\left\{i \in N \mid i \neq j_{a} \text { and } i j_{a} \notin E\right\}, J_{a}:=\left\{i \in N \mid i \neq i_{a} \text { and } i i_{a} \notin E\right\}
$$

for $a=1, \ldots, \nu$. Thus, $i_{a} \in I_{a}$ and $j_{a} \in J_{a}$ for $a \leq \nu$. Set

$$
I:=\bigcup_{a=1}^{\nu} I_{a} \cup J_{a}, N_{0}:=N \backslash I
$$

$$
S_{I}:=\{s \in S \mid s \text { is adjacent to all nodes of } I\} \text {, and } T:=S \backslash S_{I} .
$$

We group several observations about the sets $I, N_{0}, S_{I}$. By Claim $8, S_{I} \neq \emptyset$ and a node $s \in S$ belongs to $S_{I}$ if and only if $s$ is adjacent to $i_{a}$ and $j_{a}$ for some $a=1, \ldots, \nu$. Moreover,

$$
N_{0} \text { is a clique }
$$

(else we find an induced matching of size $\nu+1$ in $\bar{G}[N]$ ).

Every node of $N_{0}$ is adjacent to every node of $I$.

Indeed, suppose that $x \in I$ is not adjacent to $y \in N_{0}$; say, $x \in I_{1}$. Then, $y$ is adjacent to $i_{1}, j_{1}, i_{2}, j_{2}$ and $x \neq i_{1}$. Given $s \in S_{I}$ we find $A_{7}$ on $\left\{i_{1}, j_{1}, i_{2}, j_{2}, x, y, s, \bar{n}\right\}$. Moreover,

$S_{I}$ is a clique. 
Indeed, if $s, t \in S_{I}$ are not adjacent, then we find $A_{3}$ on $\left\{\bar{n}, s, t, i_{1}, j_{1}, i_{2}, j_{2}\right\}$. We also have:

$$
\text { If } x \in I_{a}, j \in J_{a}, a \in\{1, \ldots, \nu\} \text {, then } x y \notin E \text {. }
$$

Say, $a=1$. The statement is obvious if $x=i_{1}$ or $y=j_{1}$. Otherwise, we find $A_{7}$ on $\left\{i_{1}, j_{1}, i_{2}, j_{2}, x, y, s, \bar{n}\right\}$ where $s \in S_{I}$. The following statements (25) and (26) are direct consequences of Claim 8.

(25) If $x \in I_{a} \cup J_{a}$, then $x$ is adjacent to $i_{b}$ and $j_{b}$, for $a \neq b \in\{1, \ldots, \nu\}$.

$$
\text { If } s \in T, x, y \in I_{a} \cup J_{a} \text { and } x y \notin E \text {, then } s \text { is adjacent to }
$$
at most one of $x, y$.

$$
\begin{aligned}
& \text { If } x \in I_{a} \cup J_{a}, y \in I_{b} \cup J_{b}, a \neq b \in\{1, \ldots, \nu\}, \text { then } x y \in E \\
& \text { and } x \in\left\{i_{a}, j_{a}\right\} \text { or } y \in\left\{i_{b}, j_{b}\right\} \text {. }
\end{aligned}
$$

The statement follows from (25) if $x \in\left\{i_{a}, j_{a}\right\}$ or $y \in\left\{i_{b}, j_{b}\right\}$. Otherwise, we find $A_{4}$ on $\left\{x, y, i_{a}, j_{a}, i_{b}, j_{b}\right\}$ if $x y \notin E$ and we find $A_{8}$ on $\left\{x, y, i_{a}, j_{a}, i_{b}, j_{b}, s, \bar{n}\right\}$ if $x y \in E$ (where $s \in S_{I}$ ).

Our next objective is to show that $S=S_{I}$, i.e., $T=\emptyset$. For $s \in T$, set:

$$
Z_{s}:=\left\{x \in S_{I} \cup N \mid s x \in E\right\} .
$$

Claim 28. $Z_{s}$ is a clique for every $s \in T$ and $Z_{s} \cup Z_{t}$ is a clique for every edge st in $T$.

Proof. Suppose that $x, y \in Z_{s}$ are not adjacent for some $s \in T$. As $S_{I}$ and $N_{0}$ are cliques and every node of $I$ is adjacent to every node of $N_{0}$, there are two cases: either $x, y \in I$, or $x \in S_{I}$ and $y \in N$. The case $x, y \in I$ is excluded by relations (26) and (27). If $x \in S_{I}$ and $y \in N$, then $N(s) \cup N(y)$ is not a clique since $I \subseteq N(x)$. By Claim 2, this implies that $N(s) \subseteq N(x)$ and, thus, $x y \in E$, a contradiction.

Suppose now that $Z_{s} \cup Z_{t}$ is not a clique for some edge st in $T$; let $x \in Z_{s} \backslash Z_{t}$, $y \in Z_{t} \backslash Z_{s}$ be non adjacent. If $x, y \in N$ then $(\bar{n}, x, s, t, y)$ is an induced $C_{5}$ in $G$. Hence, $x \in S_{I}$ and $y \in N_{0}$. Consider $i_{1} \in I_{1}, j_{1} \in J_{1}$. By (26), we may assume that $s i_{1} \notin E$. Then, $t i_{1} \in E$ (else, $\left(i_{1}, x, s, t, y\right)$ is an induced $\left.C_{5}\right)$ and, thus, $t j_{1} \notin E, s j_{1} \in E$. But, then, we have found $B_{1}$ on $\left\{i_{1}, j_{1}, x, y, s, t\right\}$.

Let $A$ be a maximal subset of $T$ for which $G[A]$ is connected and the set $Z(A):=\bigcup_{a \in A} Z_{a}$ is a clique.

Claim 29. There is no edge between the sets $A$ and $T \backslash A$. 
Proof. Suppose that $a \in A$ is adjacent to $b \in T \backslash A$. By maximality of $A$, we deduce that $Z(A) \cup Z_{b}$ is not a clique; let $x \in Z(A), y \in Z_{b}$ be non adjacent and let $a_{0} \in A$ be adjacent to $x$. We know from Claim 28 that $a \neq a_{0}$ and $a_{0} y, x a, x b \notin E$. Considering a shortest path in $G[A]$ from $a_{0}$ to $a$, we find an induced path $\left(x, a_{1}, \ldots, a_{p}, y\right)$ where $a_{1}, \ldots, a_{p} \in T$ and $p \geq 3$. If $x, y \in N$, then this path together with edges $\bar{n} x, \bar{n} y$ yields an induced circuit in $G$. Hence, $x \in S_{I}$ and $y \in N_{0}$. Consider $i_{1} \in I_{1}, j_{1} \in J_{1}$. Then, $i_{1}$ is adjacent to one of $a_{1}, a_{2}$. (Indeed, $i_{1}$ is adjacent to some $a_{i}$ for, if not, then $\left(i_{1}, x, a_{1}, \ldots, a_{p}, y\right)$ is an induced circuit. Let $k \geq 1$ be the smallest index such that $i_{1} a_{k} \in E$; then, $\left(i_{1}, x, a_{1}, \ldots, a_{k}\right)$ is an induced circuit which implies that $k \leq 2$.) Similarly, $j_{1}$ is adjacent to one of $a_{1}, a_{2}$. Hence, we can assume that $i_{1} a_{1} \in E\left(\Longrightarrow j_{1} a_{1} \notin E\right), j_{1} a_{2} \in E\left(\Longrightarrow i_{1} a_{2} \notin E\right)$. Then, we find $B_{5}$ on $\left\{i_{1}, j_{1}, x, y, a_{1}, a_{2}\right\}$.

From this it follows that

$$
T=\emptyset ; \text { that is, } S=S_{I} .
$$

For, if not, then $A \neq \emptyset$ and $Z(A)$ would be a clique cutset in $G$.

Corollary 30. If $\nu=3$, or if $\nu=2$ with $\max \left(\left|I_{a} \cup J_{a}\right|: a=1,2\right) \geq 3$, then $G \in \mathcal{G}_{\nu}$.

Proof. By the assumption, we have that $|\bar{N}|=|S|=1$ and $S \cup N_{0}$ is a cliq 1e (for, otherwise, one would find $A_{8}$ or $A_{9}$ in $G$ ). Moreover, if $\nu=3$, then $\max \left(\left|I_{a} \cup J_{a}\right|: a=1,2,3\right)=2$ (else, one finds $A_{9}$ ). Using (24), (25), (27), one obtains that $G \in \mathcal{G}_{\nu}$.

Therefore, we can now assume that

$$
\nu=2 \text { and } \max \left(\left|I_{a} \cup J_{a}\right|: a=1,2\right)=2 .
$$

Set

$$
N_{0}^{\prime}:=\left\{i \in N_{0} \mid \text { is } \notin E \text { for some } s \in S\right\} .
$$

The following holds:

si $\notin E$ for every $s \in S, i \in N_{0}^{\prime}$.

Indeed, suppose that $s i \in E$ for some $s \in S, i \in N_{0}^{\prime}$ and let $t \in S$ such that ti $\notin E$. Then, we find $A_{7}$ on $\left\{\bar{n}, s, t, i, i_{1}, j_{1}, i_{2}, j_{2}\right\}$.

Hence, the node set of $G$ can be partitioned into the sets $S, N_{0} \backslash N_{0}^{\prime}, N_{0}^{\prime} \cup \bar{N}$, and $I=\left\{i_{1}, j_{1}, i_{2}, j_{2}\right\}$, in such a way that $S \cup\left(N_{0} \backslash N_{0}^{\prime}\right)$ and $\left(N_{0} \backslash N_{0}^{\prime}\right) \cup N_{0}^{\prime} \cup \bar{N}$ are cliques and there is no edge between $S$ and $N_{0}^{\prime} \cup \bar{N}$. Therefore, $G$ belongs to $\mathcal{G}_{2}$. This concludes the proof in Case $\mathrm{C}$ and, thus, of Theorem 8 . 


\section{Conclusions}

As mentioned in the Introduction, one of the motivations for the study of the cone $\mathcal{P}_{G}$ comes from its link with the positive semidefinite matrix completion problem. It has been shown in [11] that the completion problem can be solved in polynomial time for the following classes of graphs: chordal graphs (i.e., graphs with sparsity order 1); graphs with fixed minimum fill-

in; hence, for graphs in $\bigcup_{i=1}^{3} \mathcal{G}_{i}$; graphs in $\mathcal{G}_{4}$ when one of the sets on the 4-circuit is assumed to have a fixed size. Therefore, if one can show that the completion problem is polynomial time solvable over the whole class $\mathcal{G}_{4}$, then the problem would be polynomial time solvable for all graphs with sparsity order 2 .

Acknowledgements. We thank a referee for his/her careful reading of the paper and for his/her suggestions that helped improve the presentation of the paper.

\section{References}

[1] J. Agler, J. W. Helton, S. McCullough, and L. Rodman: Positive semidefinite matrices with a given sparsity pattern, Linear Algebra and its Applications, 107 (1988), 101-149.

[2] M. Deza and M. Laurent: Geometry of Cuts and Metrics. Vol. 15 of Algorithms and Combinatorics, Springer-Verlag, Berlin, 1997.

[3] G. A. Dirac: On rigid circuit graphs, Abhandlungen aus dem Mathematischen Seminar der Universität Hamburg, 25 (1961), 71-76.

[4] R. Grone, C. R. Johnson, E. M. SÁ, and H. Wolkowicz: Positive definite completions of partial hermitian matrices, Linear Algebra and its Applications, 58 (1984), 109-124.

[5] R. Grone and S. Pierce: Extremal bipartite matrices, Linear Algebra and its Applications, 131 (1990), 39-50.

[6] J. W. Helton, D. Lam, and H. J. Woederman: Sparsity patterns with high rank extremal positive semidefinite matrices, SIAM Journal on Matrix Analysis and its Applications, 15 (1994), 299-312.

[7] J. W. Helton, S. Pierce, and L. Rodman: The ranks of extremal positive semidefinite matrices with given sparsity pattern, SIAM Journal on Matrix Analysis and its Applications, 10 (1989), 407-423.

[8] R. D. Hill and S. R. WATERs: On the cone of positive semidefinite matrices, Linear Algebra and its Applications, 90 (1987), 81-88.

[9] C. R. Johnson: Matrix completion problems: a survey, in: C. R. Johnson, editor, Matrix Theory and Applications, volume 40 of Proceedings of Symposia in Applied Mathernatics, pages 171-198. American Mathematical Society, Providence, Rhode Island, 1990. 
[10] M. Laurent: Cuts, matrix completions and graph rigidity, Mathematical Programming, 79 (1997), 255-283.

[11] M. Laurent: Polynomial instances of the positive semidefinite and Euclidean distance matrix completion problems, SIAM Journal on Matrix Analysis and its Applications, 22 (2000), 874-894. (Electronic publication.)

[12] S. MCCullough: 2-chordal graphs, in: I. Gohberg, J. W. Helton and L. Rodman, eds., Contributions to Operator Theory and its Applications, Vol. 35 in Series Operator Theory: Advances and Applications, pages 143-192, Birkhäuser-Verlag, Basel, 1988.

[13] S. MCCullough: Minimal separators of 2-chordal graphs, Linear Algebra and its Applications, 184 (1993), 187-199.

[14] V. I. Paulsen, S. C. Power, and R. R. Smith: Schur products and matrix completions, Journal of Functional Analysis, 85 (1989), 151-178.

[15] D. J. Rose: Triangulated graphs and the elimination process, Journal of Mathematical Analysis and Applications, 32 (1970), 597-609.

[16] A. SCHRIJVer: A Course on Combinatorial Optimization. Lecture Notes, 1994.

[17] N. SHAKED-MONDERER: Extremal positive semidefinite matrices with given sparsity pattern. Linear and Multilinear Algebra, 36 (1994), 287-292.

[18] R. E. Tarjan: Decomposition by clique separators, Discrete Mathematics, 55 (1985), 221-232.

[19] M. Yannakakis: Computing the minimum fill-in is NP-complete, SIAM Journal on Algebraic and Discrete Methods, 2 (1981), 77-79.

Monique Laurent

CWI, P.O. Box 94079,

1090 GB Amsterdam,

The Netherlands

monique@cwi.nI 